\title{
BMJ Open Task sharing to improve the prevention, diagnosis and management of rheumatic heart disease: a systematic review protocol
}

\author{
Leila Hussein Abdullahi, ${ }^{1,2}$ Inge Smit, ${ }^{2}$ Mark E Engel, ${ }^{3,4}$ David A Watkins, ${ }^{2,5}$ \\ Liesl Joanna Zühlke $e^{2,3,4,6}$
}

To cite: Abdullahi LH, Smit I, Engel ME, et al. Task sharing to improve the prevention, diagnosis and management of rheumatic heart disease: a systematic review protocol. BMJ Open 2018;8:e019511. doi:10.1136/ bmjopen-2017-019511

- Prepublication history and additional material are available. To view these files please visit the journal online (http://dx.doi.org/ 10.1136/ bmjopen-2017-019511).

Received 11 September 2017 Revised 18 December 2017 Accepted 22 December 2017

Check for updates

${ }^{1}$ Save the Children International (SCI), Somalia/Somaliland Country Office, Nairobi, Kenya ${ }^{2}$ Department of Paediatrics, Red Cross War Memorial Children's Hospital, University of Cape Town, Cape Town, South Africa ${ }^{3}$ Department of Medicine, Groote Schuur Hospital, Cape Town, South Africa

${ }^{4}$ Faculty of Health Sciences, University of Cape Town, Cape Town, South Africa

${ }^{5}$ Division of General Internal Medicine, University of Washington, Seattle, Washington, USA

${ }^{6}$ Division of Cardiology, Groote Schuur Hospital, Cape Town,

South Africa

Correspondence to Dr Liesl Joanna Zühlke; liesl.zuhlke@uct.ac.za

\section{ABSTRACT}

Introduction Rheumatic heart disease (RHD) remains an important cause of cardiovascular morbidity and mortality globally due to weak health systems in many countries. RHD can be effectively prevented and managed; however, RHD-related interventions have not been widely adopted in countries with severe human resource constraints. Task sharing is a recognised approach to personnel shortages that could prove effective for RHD and has, anecdotally, been attempted in a few settings. We propose a systematic review and meta-analysis protocol to assess models that use non-physician workers to expand access to, and quality of, RHD-related healthcare.

Methods We will include randomised controlled trials (RCT), cluster RCTs, quasi-experimental and controlled before and after studies providing information on the effectiveness of non-physician workers in providing care for streptoccocal pharyngitis, rheumatic fever and RHD. We will search relevant electronic databases and grey literature using medical subject headings. Standardised data extraction forms will be used to collect effect sizes that will be pooled in random-effects models. We will also conduct subgroup analyses and note other important quantitative findings, such as cost reduction, and qualitative findings, such as patient satisfaction. We will also assess study quality and risk of bias and metabias. Ethics and dissemination Ethics approval is not required for this systematic review of previously published literature. The results of the systematic review will be broadly disseminated via conference presentations, multidisciplinary workshops and peer-reviewed publications.

PROSPERO registration number CRD42017072989.

\section{INTRODUCTION}

Rheumatic heart disease (RHD) remains a major cause of morbidity and mortality in low/middle-income countries. ${ }^{12}$ It is a chronic heart valve disease that results from untreated streptococcal pharyngitis infections that lead to acute rheumatic fever (ARF) and RHD. Over the past few decades, RHD mortality has declined in high-income countries and a number of middle-income countries, owing both to
Strengths and limitations of this study

- This is the first systematic review protocol that has investigated the effectiveness of task shifting/sharing in the prevention, diagnosis and management of rheumatic heart disease (RHD).

- Our protocol will potentially review a wide range of studies that assess the effects of task-sharing interventions.

- We propose a flexible, logic model based approach to data extraction and synthesis, which is essential since the actual intervention designs are likely to vary significantly across settings and by type of care (prevention vs treatment, etc).

- The review will be limited by including only articles in English.

- The findings of this study could inform policy and practice by providing guidance on best practices in task shifting/sharing in the prevention, diagnosis and management of RHD. At a minimum, the study will identify a research agenda in this area.

improvements in living conditions (ie, reduced transmission of streptococcal infections) and to the scale-up of prevention and treatment interventions in healthcare settings. ${ }^{34}$ Nevertheless, RHD remains a major problem in many parts of the world. In 2015, there were approximately 320000 deaths from RHD and 33 million individuals living with RHD. ${ }^{5}$ In sub-Saharan Africa, South Asia and Oceania, the overall prevalence of RHD was estimated at $10-15$ per $1000 .^{6-8}$

RHD can be prevented by means of community-based programmes that increase awareness and early detection of sore throat, ARF and RHD. ${ }^{9}$ According to WHO, successful prevention of ARF and control of RHD involves a multipronged strategy that includes disease-specific education, treatment of streptococcal infection (primary prevention), provision of long-term penicillin to individuals who have a history of ARF or RHD (secondary prevention), and medical 
and surgical treatment (tertiary prevention) of patients with complications of RHD. ${ }^{2}$ However, these prevention programmes/interventions are not well implemented in many low/middle-income countries due to severe human resource shortages, particularly for chronic non-communicable diseases like RHD.

A widely proposed solution to health workforce shortages is task sharing, sometimes called task shifting. Task shifting refers to the redistribution of tasks among health workforce teams, often from a few, highly trained health providers to a larger contingent of providers with less formal healthcare training. ${ }^{10}$ Task sharing is allowing a wider range of cadres to offer certain services when this can be done safely and effectively. As such, task sharing is a means of rapidly expanding access and improving healthcare. ${ }^{11}$ Task shifting and/or sharing have already been implemented in a number of countries with health workforce crises. Examples of HIV task shifting include provision of Anti-retrovirals (ARVs) by nurses and midwives and HIV testing and counselling by lay health workers (LHWs). (In this review, we will use task sharing to imply both task-shifting and sharing activities.)

There is evidence that task sharing has made a positive contribution to other disease programmes like HIV/AIDS, family planning and overall health systems strengthening. ${ }^{12}$ While there is a paucity of evidence on the task sharing to address RHD, the use of task sharing in principle could be applied to this condition, for example, through the effective use of LHWs to support chronic disease management. ${ }^{13}$ Our systematic review aims to provide contemporary information on models of care that use non-physician workers to expand access to care and/or improve the quality of care for strep throat, ARF and RHD.

\section{OBJECTIVE}

To investigate the effectiveness of task sharing in the prevention, diagnosis and management of RHD.

\section{METHODS}

\section{Criteria for considering studies for this review}

The protocol was prepared according to the Preferred Reporting Items for Systematic review and Meta-Analysis (PRISMA) guidelines. A PRISMA Protocol checklist is completed and included in the online supplementary appendix $1 .^{14}$

\section{Types of studies}

Randomised controlled trials, cluster randomised controlled trials, quasi-experimental studies, controlled before and after studies and case reports.

\section{Types of participants}

For studies where the unit of analysis is the patient, we will include studies where participants are at risk of, or affected by strep throat, ARF and RHD. For studies where the unit of analysis if the healthcare worker, we will include studies where participants are tasked with providing care for strep throat, ARF and RHD.

\section{Interventions}

We define 'interventions' as any measures that seek to share/shift any tasks related to RHD prevention or management in healthcare facilities or relevant community settings (such as schools).

\section{Controls}

We define 'controls' as the standard(s) of care relevant to the clinical scenario. Usually, this will be a specialist or generalist physician, though in some cases (such as sore throat treatment) the standard may be a clinical officer or primary care nurse.

\section{Types of outcome measures}

We will assess study outcomes using a logic model framework applied to the three major levels of RHD care: primary, secondary and tertiary prevention (table 1 ). The use of logic models is appropriate in the evaluation of health system interventions where long-term health impact of an intervention can be difficult to quantify and processes (and intermediate outcomes) are important from a policy and planning standpoint. In advance of conducting the review, we do not have sufficient detail on the types of outputs, outcomes and impacts that studies report. Examples of study outcomes are listed in table 1 below.

\section{Searches}

A comprehensive search strategy will be developed to search both published and unpublished articles, with restrictions to English language but no restriction on the publication date. We will include Medical Subject Headings and free-text terms relating to RHD and various strategies of task sharing/shifting, such as training of

\begin{tabular}{llll}
\hline Table 1 & Matrix of potential outcomes & & \\
\hline Prevention levels & Example output & Example outcome & Example impact \\
\hline Primary & $\begin{array}{l}\text { Number of tests for group A } \\
\text { streptococcus performed }\end{array}$ & $\begin{array}{l}\text { Time spent per patient visit for } \\
\text { sore throat }\end{array}$ & $\begin{array}{l}\text { Number of cases of strep throat } \\
\text { effectively treated (cured) }\end{array}$ \\
Secondary & $\begin{array}{l}\text { Number of nurses trained in safe } \\
\text { penicillin administration }\end{array}$ & $\begin{array}{l}\text { Proportion of months (or patients) } \\
\text { adherent to secondary prevention }\end{array}$ & $\begin{array}{l}\text { Number of ARF recurrences (per } \\
\text { individual or in population) }\end{array}$ \\
Tertiary & $\begin{array}{l}\text { Number of referrals for RHD } \\
\text { placed }\end{array}$ & $\begin{array}{l}\text { Time in therapeutic range } \\
\text { (international normalised ratio) for }\end{array}$ & $\begin{array}{l}\text { Number (or rate) of hospital } \\
\text { admissions for RHD complications }\end{array}$ \\
\hline
\end{tabular}

ARF, acute rheumatic fever; RHD, rheumatic heart disease. 
healthcare workers in low/middle-income countries (table 2). The developed search strategy will be adapted to suit each individual database using applicable controlled vocabulary. We will screen the following electronic databases: PubMed, Cochrane Central Register of Controlled Trials, Scopus, Web of Science and Cumulative Index to Nursing and Allied Health Literature.

In addition, we will search for conference proceedings from XIX Lancefield International Symposium on Streptococci and Streptococcal disease. Google Scholar and the WHO Library Information System website will be searched for grey literature. We will hand-search reference lists of the key articles retrieved during the search.

\section{Selection of studies for inclusion}

Two authors will independently screen the search outputs (titles and abstracts) to select potentially eligible studies, and then review the full text of potentially eligible studies. A third author will resolve discrepancies. We will use a PRISMA flow chart to summarise the search and selection of studies for the review. We will include a table of all included studies in the review and document the reasons for exclusion of studies.

\section{Data extraction and management}

We will use a standardised/adapted data extraction form to independently extract information from included articles. This data extraction process will be duplicated independently in order to improve reliability. The data extraction form will capture (1) basic study characteristics, including objectives, study population, sample size, years and location of study and study design; (2) details of intervention(s) and study outcomes (organised according to the matrix in table 1) and (3) an open-ended section for other important quanitative findings (such as reductions healthcare costs) and qualitative findings (such as patient satisfaction) (online supplementary appendix 2). Where study data are unclear, the original author of the manuscript will be contacted to clarify his or her findings.

\section{Assessment of risk of bias in included studies}

All selected studies will be critically appraised in terms of their design methodology, biases and confounders. Cochrane Collaboration tool (ROB 2.0) will be used to assess the risk of bias of randomised control trial studies. Risk of Bias tool consist of five standard criteria: bias arising from the randomisation process, bias due to deviations from intended interventions, bias due to missing outcome data, bias in measurement of the outcome and bias in selection of the reported result. ${ }^{15}$ The other study designs will be assessed using the Critical Appraisal Skills Programme checklist. ${ }^{16}$ For each included study, we will report our assessment of risk of bias together with a descriptive summary of the information that influenced our judgement. We will judge the 'Risk of Bias' in an included study as either 'low risk', 'high risk' or 'unclear risk'. We will present a 'Risk of bias' figure to summarise these assessments. Two authors will resolve disagreements in the assessment of risk of bias by discussion and consensus, consulting a third reviewer to resolve any persistent disagreements.

\section{Dealing with missing data}

Corresponding authors of included studies will be contacted for missing data when necessary. Failing to get a response from the corresponding author, other authors will be contacted copying the corresponding author. Sensitivity analyses will be performed to assess the extent to which missing data could affect the primary outcome.

\section{Assessment of heterogeneity}

If studies of similar interventions reporting similar outcomes are included, statistical heterogeneity will be examined using the $\chi^{2}$ test for homogeneity (with significance defined at $10 \%$ alpha level). Statistical heterogeneity will be quantified using the $\mathrm{I}^{2}$ statistic. ${ }^{15}$

\section{Data synthesis}

The outcomes of interest will be either dichotomous or continuous; we will calculate risk ratios and their corresponding 95\% CI and $\mathrm{P}$ values for dichotomous outcomes, and mean differences and SD for continuous outcomes. Where outcomes are measured using different scales, we will calculate standardised mean differences. If studies are sufficiently homogenous (relating to study populations, interventions and outcomes), then data will be pooled across studies and estimated summary effect sizes will be computed using random effects models. If meta-analysis is not feasible, the findings will be presented in narrative form, organised in matrices similar to table 1 . The study summary will include relevant tables and figures to aid in data presentation.

\begin{tabular}{|c|c|}
\hline Subject & Search terms \\
\hline Task shifting & $\begin{array}{l}\text { (task shift*(tiab) OR task-sharing *(tiab) OR balance of care(tiab) OR non-physician clinician*(tiab) OR } \\
\text { nonphysician clinician*(tiab) OR task sharing(tiab) OR community care giver*(tiab) OR community } \\
\text { healthcare provider*(tiab) OR cadres(tiab) OR 'Community Health Workers'(Mesh)) }\end{array}$ \\
\hline GAS, RHD and ARF & $\begin{array}{l}\text { (('Pharyngitis'(MeSH) OR pharyngitis OR sore throat OR strep OR group a } \beta \text {-hemolytic streptococcal } \\
\text { OR 'streptococcus pyogenes'(MeSH) OR group a streptococcus OR group a streptococcal infection } \\
\text { OR 'impetigo' (MeSH) OR impetigo OR group a streptococcus skin infection OR rheumatic fever OR } \\
\text { 'rheumatic fever' (MeSH) OR rheumatic heart disease OR 'rheumatic heart disease' (MeSH))) }\end{array}$ \\
\hline
\end{tabular}

ARF, acute rheumatic fever; GAS, Group A streptococcus; MeSH, Medical Subject Headings; RHD, rheumatic heart disease. 


\section{Subgroup analysis}

Subgroup analyses may be conducted if possible, taking into account but not limited to: types of healthcare workers, study design, level of prevention and study setting (world region or country income level).

\section{Sensitivity analysis}

If studies are similar enough to justify meta-analysis, sensitivity analyses will be conducted as appropriate to check the robustness of the results.

\section{Grading the quality of evidence}

The Grading of Recommendations Assessment, Development and Evaluation (GRADE) approach will be used to assess the quality of evidence related to each of the primary outcomes. The GRADE approach will assess the quality of evidence as very low, low moderate or high, with implications on how confident we can rely on the results and if further research is likely to change the results.

\section{Assessment of reporting biases}

We will search for and include relevant unpublished studies to reduce possible publication bias. In addition, publication bias will be assessed using symmetry of funnel plots if 10 or more eligible studies are identified.

\section{DISCUSSION}

We anticipate that this review will not only be useful in giving evidence on the expanding access to care and/or improve the quality of care for strep throat, ARF and RHD but also it will inform the policy-maker on the role and potential impact of task sharing. A task sharing policy will promote rational redistribution of tasks among existing health workforce cadres. It will allow moving specific tasks, where appropriate, from highly qualified health workers to health workers with shorter training and fewer qualifications in order to make more efficient use of the available health workers and to improve access to services. We hope that lessons for successful implementation in different contexts will also be identified.

\section{Reporting of this review}

The eligibility criteria of studies and the selection process of relevant articles will be summarised as flow diagrams. This systematic review will be reported according to the PRISMA guidelines. ${ }^{14}$

\section{ETHICS AND COMMUNICATION}

No formal ethical review is required, as the systematic reviews will use publicly available data. The findings of this systematic review will be broadly disseminated via conference presentations, multidisciplinary workshops and peer-reviewed publications.

Contributors LJZ conceived the study idea and all the authors contributed to the conception and design of the protocol. LHA wrote the first draft and all authors edited the subsequent versions of the draft. All authors (LHA, LJZ, IS, DAW and MEE) have reviewed and accepted the final version of the protocol and have given their permission for publication. LHA, IS, DAW and LJZ will perform the literature searches while LHA and IS will extract data and LHA and MEE will conduct the data analysis. All authors (LHA, LJZ, IS, DAW and MEE) are in agreement to be accountable for all aspects of the work in ensuring that questions related to the accuracy or integrity of any part of the work are appropriately investigated and resolved.

Funding LJZ, DAW and LAH received funding from Medtronic Foundation through support to RHD Action. LJZ and IS receive funding from the NRFSA (National Research Foundation of South Africa)

Competing interests None declared.

Patient consent Not required.

Provenance and peer review Not commissioned; externally peer reviewed.

Open Access This is an Open Access article distributed in accordance with the Creative Commons Attribution Non Commercial (CC BY-NC 4.0) license, which permits others to distribute, remix, adapt, build upon this work non-commercially, and license their derivative works on different terms, provided the original work is properly cited and the use is non-commercial. See: http://creativecommons.org/ licenses/by-nc/4.0/

(c) Article author(s) (or their employer(s) unless otherwise stated in the text of the article) 2018. All rights reserved. No commercial use is permitted unless otherwise expressly granted.

\section{REFERENCES}

1. Mirabel M, Narayanan K, Jouven X, et al. Prevention of acute rheumatic fever and rheumatic heart disease. Circulation 2014;130:e35-37.

2. Remenyi B, Carapetis J, Wyber R, et al. Position statement of the World Heart Federation on the prevention and control of rheumatic heart disease. Nat Rev Cardiol 2013;10:284-92.

3. Carapetis JR, Steer AC, Mulholland EK, et al. The global burden of group A streptococcal diseases. Lancet Infect Dis 2005;5:685-94.

4. Marijon E, Mirabel M, Celermajer DS, et al. Rheumatic heart disease. The Lancet 2012;379:953-64.

5. Watkins DA, Johnson CO, Colquhoun SM, et al. Global, regional, and national burden of rheumatic heart disease, 1990-2015. N Engl J Med 2017;377:713-22.

6. Beaton A, Okello E, Aliku T, et al. Latent rheumatic heart disease: outcomes 2 years after echocardiographic detection. Pediatr Cardiol 2014;35:1259-67.

7. Vos T, Barber RM, Bell B, et al. Global, regional, and national incidence, prevalence, and years lived with disability for 301 acute and chronic diseases and injuries in 188 countries, 1990-2013: a systematic analysis for the Global Burden of Disease Study 2013. Lancet 2015;386:743-800.

8. Karthikeyan G, Mayosi BM. Is primary prevention of rheumatic fever the missing link in the control of rheumatic heart disease in Africa? Circulation 2009;120:709-13.

9. Zuhlke L. Rheumatic heart disease and the ASAP programme: Fresh insights into an old disease. CME 2011;29:460-2.

10. WHO/UNAIDS/PEPFAR. Task shifting: global recommendations and guidelines. http://www.who.int/healthsystems/task_shifting/en/index. html

11. World Health Organization (WHO). The World Health Report 2006Working together for health. http://www.who.int/whr/2006/whr06_en. pdf

12. Callaghan $\mathrm{M}$, Ford $\mathrm{N}$, Schneider H. A systematic review of taskshifting for HIV treatment and care in Africa. Hum Resour Health 2010;8:8.

13. Lewin SA, Dick J, Pond P, et al. Lay health workers in primary and community health care. Cochrane Database Syst Rev 2005:CD004015.

14. Moher D, Shamseer L, Clarke M, et al. Preferred reporting items for systematic review and meta-analysis protocols (PRISMA-P) 2015 statement. Syst Rev 2015;4:1-9.

15. Higgins JPT, Green S, eds. The Cochrane Collaboration. In: Cochrane Handbook for Systematic Reviews of Interventions Version 5.1.0 [updated March 2011], 2011. http://handbook.cochrane.org

16. Critical Appraisal Skills Programme (CASP) (2014) CASP research checklist. Oxford. http://www.casp-uk.net/casp-tools-checklists (accessed May 2017). 